\title{
Female Urethroplasty: A new hope for better quality of life
}

\author{
Md. Zahid H Bhuiyan', Syed Alfesani ${ }^{2}$
}

Received: 19 - 07 - 2020

Accepted: 11 - 09 - 2020

Conflicts of interest: None

Keywords: Female urethral stricture, urethral instrumentation, VCUG, OMG urethroplasty

\begin{abstract}
Introduction: Female urethral stricture is a difficult issue in Urology. Many of the times it may follow any sort of urethral instrumentation but in other cases no definite precipitating causes are unidentified.

Methods: This was a prospective study. The sample size was $n-50$. All cases were recurrent with the history of urethral dilatations, urethrotomies in multiple sessions. The diagnostic investigations in VCUG (voiding cystourethrogram), Ultrasound scan of kidney Ureter, bladder with post void residues, Uroflometry etc. Due to limitations, Urodynamic study was done in limited cases. Urethral augmentation was done using dorsal substitution of OMG (oral mucosal graft). Urethral catheter was left for $03-04$ weeks.
\end{abstract}

Results: After removal of catheter, urinary flow was observed; excellent in all the cases. It remain satisfactory in 06 months of follow-up. Overall result is excellent.

Conclusions: Female urethral stricture is a difficult issue in Urology, Urethral dilatation, urethrotomies, clean intermittent catheterization are good options but need frequent procedures. Urethral augmentation with dorsal substitution of oral mucosal graft is a curative approach in long-run.

\section{Introduction:}

Bladder outlet obstruction, of which true urethral stricture is only a minor component in women ${ }^{1}$. Still female urethral stricture diseases is a difficult issue in the Urological practice. Women. are silent sufferer. In long run there may be progressive deterioration of bladder and renal function.

Due to high PVR frequency may be a predominating symptom. Which may further worse with anticholinergic drugs, used to reduce frequency? Recurrence is common by traditional treatment modalities. However curative treatment may be possible by urethroplasty ${ }^{2}$.

Female urethral stricture may be caused by urethral instrumentation like catheter placement prior any surgery,. cystoscopy with or without urethral dilation, sling surgery for urinary incontinence. So the most common causes are therefore iatrogenic in origin. infection, trauma may be other causes, Although true urethral stricture disease (urethral tissue fibrosis/ scarring leading to luminal narrowing) is rare, dilation is commonly and sometimes inappropriately

1. Professor and Head, Department of Urology, Bangladesh Medical College and Hospital, Dhaka.

2. Assistant Professor, Department of Urology, Jamalpur Medical College and Hospital, Dhaka.

Correspondences: Prof. Dr. Md. Zahid H Bhuiyan, Professor and Head, Department of Urology, Bangladesh Medical College and Hospital, Dhaka. E-mail: zahidhb_1961@yahoo.com 
performed $^{3}$. Dilation may thus create more strictures than it treats.

\section{Methods:}

The study was prospective, done in the department of Urology, Bangladesh Medical College and hospital and some private clinics. The study period was from January'2010 to December'19.The sample size was n 50. To findout the aetiology proper history, physical examination, and allied investigations were taken. In $\mathrm{n}-26$ cases, had history of LUCS (Lower uterine caesarian section) and simultaneous urethral catheterization, n - 03 cases were underwent cystoscopic evaluation for LUTS, in their premanoposal age. All stricture cases were recurrent, received multiple urethral dilatation and or urethrotomies, In $\mathrm{n}-05$ cases were admitted with the history of acute on chronic retention and SPC (suprapubic catheter) in situ.The SPC was unrelated to menopause ( $\mathrm{n}-03$ premenopausal, $\mathrm{n}-02$ postmenopausal).urethroplasty wasdone after keeping SPC in situ for three months, aiming to rest the bladder to regain its function following long period of chronic retention. Out of $n-50$ cases; $n-16$ cases are postmenopausal, and $\mathrm{n}-34$. casesare premenopausal. In all cases negative urine $\mathrm{C} / \mathrm{S}$ was ensured before operation. Urodynamic study was done in $n-05$ cases of SPCs through suprapubic catheter channel. Those appears equivocal. In other cases $(n-45)$; Ultrasound scan reported thick bladder wall high PVR ( post voidresidue), stricture patern of Uroflowmetry, suggestive of increase bladder activity. MCU (Micturating cystourethrogram) was done only in $\mathrm{n}-$ 08 doubtful cases. The typical finding was dilated bladder neck and proximal urethra and distal urethral and meatal narrowing. Our protocol was to use free Buccal Musosal Graft (BMG) after harvesting it from oral vestibule, below the level of papilla of parotid duct.

Dorsal inlay urethroplasty was done in all cases by a single surgeon. We have counseled the patients and party in details before surgery. In some cases urethra was so narrow to accommodate a narrowest feeding tube ( $5 \mathrm{fr}$ ) or $.035 \mathrm{~mm}$ glide wire. In such cases we introduce gulide wire through stricture, cystoscopically. Dorsal internal urethrotomy was done for widening the urethra $(n-19)$.

Initially a plane of dissection was planned by a inverted semi-circular incision; around upper half of urethral meatus between urethra and clitoris after injecting. $01 \%$ adrenaline along the plane of dissection. Urethra then dissected and split dorsally, extending proximally from meatus; $1 \mathrm{~cm}$ beyond the stricture segment. By this time buccal mucosa was harvested from oral vestibule either under L/A (local anaesthesia) n - 35 or G/A (general anaesthesia) $n-15$, depending on patient's compliance. After defating the buccal mucosal free graft substitution was so designed, to reconstruct the urethral caliber to accommodate $18 \mathrm{fr}$ catheter which fit snugly. Buccal mucosal anchoring started from the apex of the split urethra, and tucked to the underlying tissue and subsequently to the body of the clitoris using $4^{\prime} 0^{\prime}$ vicryl. Both margins of free graft anchored to urethral margin with underlying tissues. At the end of Urethroplasty we replace the $18 \mathrm{Fr}$. Catheter by $14 \mathrm{Fr}$. Dilute povidone iodine socked gauze wrapped twice around the catheter with a single knot, pushed proximally upto the meatus, to ensure wet graft margin at the meatus. A vaginal pack also kept to stabilize the grafted urethra. Both the pack and gauge were kept for 48 hours and started urethral toileting around the catheter using povidone iodine ointment.

The urethral catheter was kept for 03 weeks. Normal voiding observed after removal of catheter, confirmed by Uroflometry.

Table I. Distribution of age. The affected women are unrelated to menopausal age $(n-50)$,

\begin{tabular}{lccc}
\hline Age in years & minimum & maximum & mean \\
& 35 & 59 & 44 \\
\hline Premanoposal $(\mathrm{n}-34)$ & 35 & 44 & 41 \\
Postmanoposal $(\mathrm{n}-16)$ & 48 & 59 & 54 \\
\hline
\end{tabular}

Table II: (05 is on SPC) Analysis of Uroflometry. The preoperative uroflometry was severely obstructed pattern $n-45$

\begin{tabular}{lccc}
\hline Q max (maximum flow rate) & Highest $: 09 \mathrm{ml}$ & Lowest $: 04 \mathrm{ml}$ & Mean : 06 ml \\
Q mean (minimum flow rate) & Highest $: 06 \mathrm{ml}$ & Lowest $: 02 \mathrm{ml}$ & Mean : 04 ml \\
voided volume $>150 \mathrm{ml}$. & Maximum $: 680 \mathrm{ml}$ & Minimum $: 150 \mathrm{ml}$ & Mean : $280 \mathrm{ml}$ \\
\hline
\end{tabular}


Table III : ( $\mathrm{n}-45,05$ is on SPC) probable causes. History of urethral instrument is only on $n-29$ cases.

\begin{tabular}{lc}
\hline Urethral catheterization & $\mathrm{n}-26$ (LUCS) \\
\hline Diagnostic cystoscopy & $\mathrm{n}-03$ \\
No history of any urethral & $\mathrm{n}-21$ \\
instrumentation & \\
\hline
\end{tabular}

\section{Result:}

Urine flow is excellent after removal of catheter. Uroflometry and USG done after 06 weeks. Even many of the patients $(n-12)$ become supervoider. The data of total $(n-50)$ number of patients before and after Urethroplasty has been described in tabulated form and comperative picture to show the operative outcome

Table IV: $(n-50)$ Analysis of Post - operative Uroflometry revealed $n-12$ cases are supervoider in Group A, Excellent in Group B ( $n-33$ cases). Reasonably normal in $(n-05)$ in Group C.

\begin{tabular}{lccc}
\hline Group A $(\mathrm{n}-12)$ & Q max $: 55 \mathrm{ml}$ & Mean $: 18 \mathrm{ml}$ & Supervoider \\
Group B $(\mathrm{n}-33)$ & Q max $: 32 \mathrm{ml}$ & Mean $: 12 \mathrm{ml}$ & Excellentl \\
Group C $(\mathrm{n}-05)$ & Q max $: 18 \mathrm{ml}$ & Q mean $: 09 \mathrm{ml}$ & Good \\
Voided volume $>150 \mathrm{ml}$. & Maximum $: 650 \mathrm{ml}$ & Minimum $: 450 \mathrm{ml}$ & Mean : $520 \mathrm{ml}$ \\
\hline
\end{tabular}

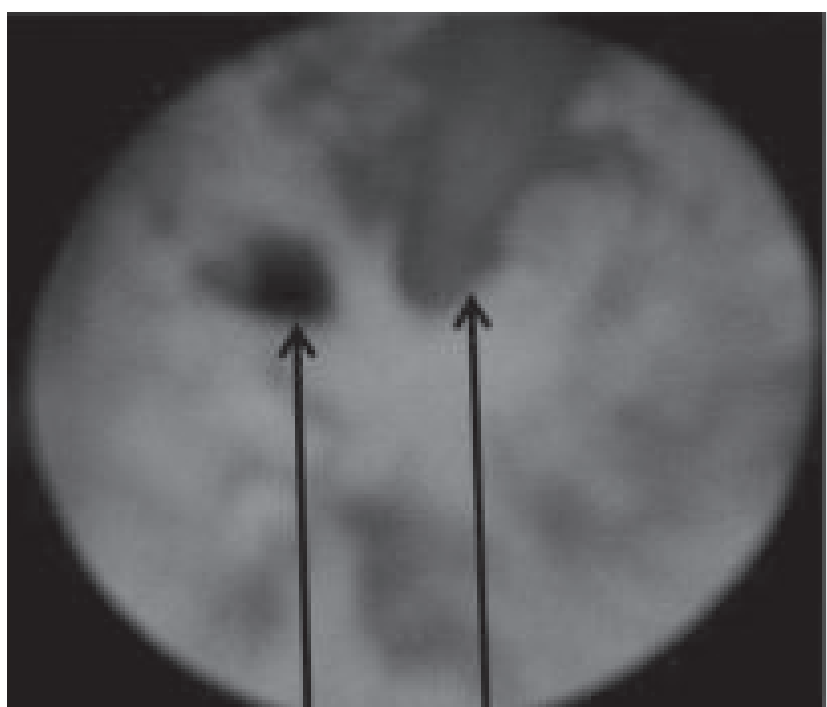

Stricture accomodating a guide wire Adjacent orifice is a false passage

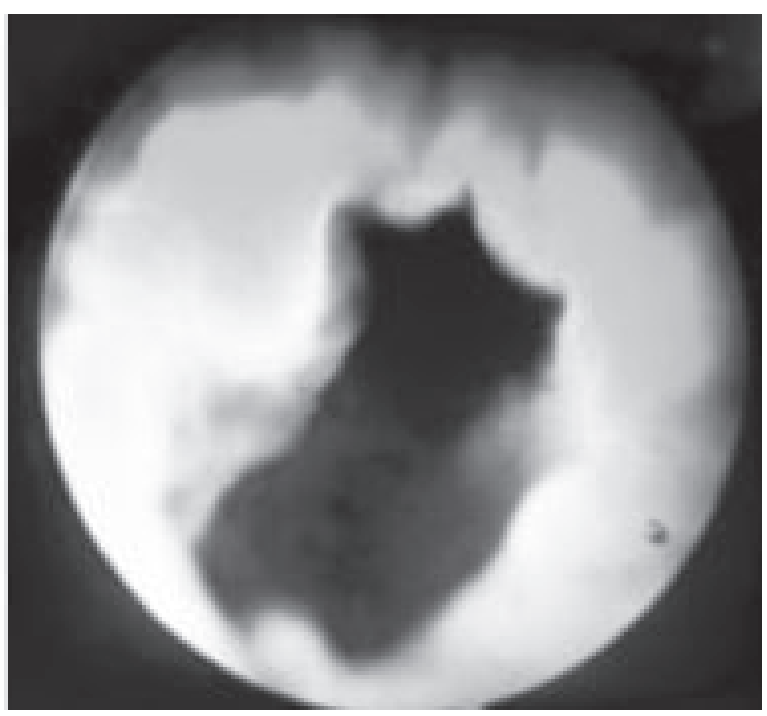

Post operative view :

After urethroplasty

Fig.-1: Cystoscopic view before and after reconstruction

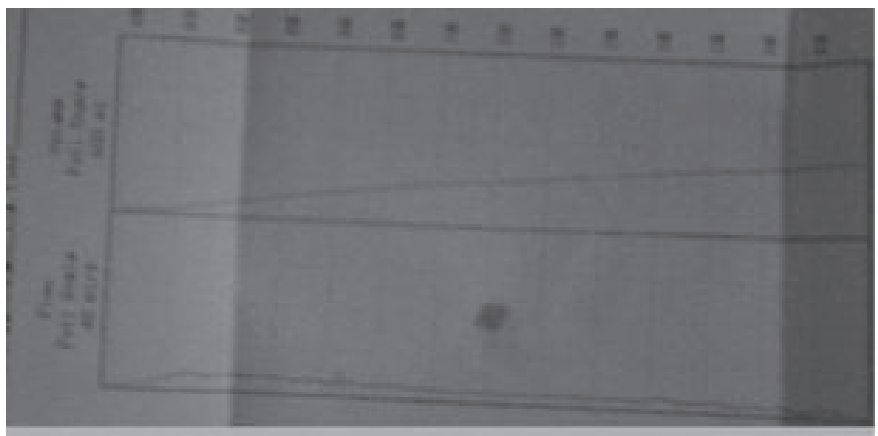

Uroflowmetry

Q max: 5ml/sec, Mean: $2 \mathrm{ml} / \mathrm{sec}$. Voided volume: $250 \mathrm{ml}$

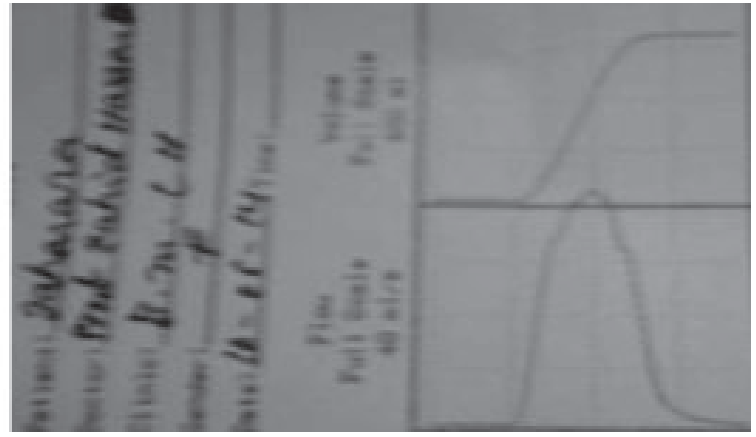

Uroflowmetry

Q max: 58ml, Mean : $31 \mathrm{ml}$

Fig.-2: Comparison between pre-poerative uroflometry 
All patients were followed for at least 06 months, monthly with urine R/ E, C/S, Uroflowmetry, USG of KUB + PVR. The Overall result was excellent.

\section{Discussion:}

Female urethral stricture as a cause of bladder outlet obstruction is a rare entity. It has been estimated that only $3-8 \%$ of women who present to urologists with voiding complaints have a diagnosis of outlet obstruction ${ }^{1}$.

Out of them only subset of obstruction requiring urethral stricture management, ranges from $4 \%$ to $13 \%$. So the true incidence of urethral stricture in women presenting with voiding complaints would be $0.1-1 \%$ at greatest estimation ${ }^{2}$.

In our country the true incidence of urethral stricture is not known but it is less common in our clinical practice.

A diagnosis of urethral stricture is difficult to make based on symptomatology alone, as voiding symptoms correlate poorly with the presence of urethral obstruction in women ${ }^{4}$.. Frequency and urgency are highly nonspecific. A voiding diary may help clarify the nature of the patient's voiding complaints and reveal less common symptoms of dysuria, hesitancy, dribbling, incontinence, recurrent urinary tract infections, or pain with micturition ${ }^{5}$. History positive for prior pelvic trauma, pelvic surgery, or urethral dilations may suggest stricture, the diagnosis typically requires a combination of endoscopic and radiologic studies $^{6}$.

But the combination of urodynamics with imaging, or in conjunction with a voiding cystourethrogram, may provide additional information about stricture location and length. Failure of the bladder neck and proximal urethra to open upon initiation of detrusor contraction suggests proximal bladder outlet obstruction, not urethral stricture. The classic "spinning top" appearance of the urethra on voiding with an open, dilated proximal urethra and narrowed area at the meatus, As stricture is typically located at the distal third of the urethra or the meatus, imaging may reveal ballooning of the bladder neck on voiding.. Many women find it difficult to void in a standing position, and clean images are difficult to obtain in the seated position. When a voiding cystourethrogram is nondiagnostic, cystoscopy generally is confirmatory. Cystoscopy combined with urethral calibration using bougie à boule can help determine the caliber of the strictured urethra. Some strictures are apparent at cystoscopy as circumferential scars, similar to urethral strictures in men. In others, the patient cannot relax, and external urethral sphincteric contraction can make it difficult to visualize the stricture directly ${ }^{6,7}$.

In our series all cases were recurrent stricture with past documents of multiple interventions, namely urethral dilatations and urethrotomies. However we reviewed all the patients again. We did urine routine examinations and culture in all the cases and ensured negative culture before intervention. All of our patients were underwent ultrasound scan. Upper tract were normal in all the cases. Bladder wall thickness was more than normal $(>3 \mathrm{~mm})$; suggesting thickened detrusor with mucosal oedema. Abnormally high PVR was evaluated in $n-45$ cases (other than $n-05$ cases with SPCs). We did uroflometry in $\mathrm{n}-45$ cases; suggestive of severe outflow obstruction. We did urodynamic study in $n-05$ cases (those presented with SPC in situ) through suprapubic catheter route. The results were equivocal and we delayed urethral reconstruction for three months to ensure rest of the weak detrusor. We did imaging study (MCU) in $n-08$ cases, which were diagnostic. We perform urethroscopy in all cases before intervention in the same session; which were diagnostic. In $n-45$ cases the urethra is so narrow, necessitate dorsal urethrotomy to avoid blind dilatation to obtain clear margin of urethra during urethroplasty.

Female urethral stricture is typically iatrogenic, resulting from prior urethral dilations, difficult/ traumatic catheterization with subsequent fibrosis, urethral surgeries (diverticulum surgery, fistula repair, anti-incontinence procedures), trauma (pelvic fracture), pelvic radiation, or acute/chronic urethritis/ cystitis leading to fibrosis. There have been rare reports of urethral stricture resulting from urethral tuberculosis ${ }^{8}$. vulvar dystrophy, lichen sclerosis ${ }^{9}$. primary urethral carcinoma, urethral fibroepithelial polyps, urethral leiomyoma ${ }^{10}$. bladder drainage of pancreatic transplants, post-transurethral resection of a bladder tumor, or transsexual reconstruction ${ }^{11}$.

In our series $n-26$ cases had history of catheterization during LUCS, $\mathrm{n}-03$ cases were underwent diagnostic cystoscopy. No significant causes are found in history, as the aetiology of stricture.

Once a diagnosis of urethral stricture is made, surgical intervention is the appropriate treatment. Management depends on stricture type, location, severity, length, 
patient preference. Most female urethral strictures are treated with office based urethral dilatation in the western world but in difficult cases, serial urethral dilation or internal urethrotomy. However, the stricture-free interval after dilation or internal urethrotomy is variable, and with time, almost all strictures will recur. No conclusive clinical evidence suggests that in long run this procedure has any curativebenefit in this disease ${ }^{12}$.Patients interested in definitive management with better long-term outcomes will need open reconstructive surgery.

The evidence for urethral dilation and urethrotomy for true stricture disease is made up primarily of case reports and small series of patients with minimal follow-up ${ }^{13}$.

A prospective, randomized trial compared urethral dilation with sounds and dilatation followed by CISC (clean intermittent self catheterization) for the management of recurrent urethral strictures. Patients maintained on CISC had better objective urinary flow rates, less incidence of UTI and better subjective quality-of-life scores ${ }^{14}$.

Given the subjective responses noted in these studies, an initial trial of conservative treatment may be reasonable in selected patients with mild complaints. For most patients, with short period of recurrent; however, definitive surgical treatment will be needed. Considering high recurrence rate, the specific approach to urethroplasty should be tailored to each patient, with additional consideration of the location, length of the stricture, and integrity of the bladder neck ${ }^{15}$.

As all the patients were recurrent cases, after failure of dilatation and urethrotomies in multiple sessions our patients have only choice of urethroplasty.

A solid understanding of urethral anatomy is necessary to expose the diseased urethral segment completely while avoiding damage to surrounding structures.

The erectile tissue of the clitoris supports the urethra dorsally. The neurovascular bundles of the clitoris run along the ischiopubic ramus, below the pubic ramus in the midline. They coalesce to travel along the cephaloid surface of the clitoral body toward the glans, away from dorsal dissection planes.

The principles of female urethral reconstruction differ from those in men. In women, excision and reanastomosis of an entire urethral stricture segment is substantially more morbid and may risk stress incontinence.
A ventral approach may be more comfortable for most surgeons, as development of a plane ventral to the urethra is more frequently performed in other vaginal procedures, and minimal to no urethral mobilization is required. A dorsal approach leaves the anterior vaginal wall untouched, minimizing any potential complications that might occur with later sling placement. This approach may preserve the normal anatomic positioning of the urethra upward, whereas ventral approaches tend to redirect the urethral meatus posteriorly ${ }^{16}$.

Flap-based urethroplasty techniques have demonstrated efficacy and safety and are the treatment of choice for most female urethral strictures. Gormley recently reported durability with very low morbidity using simple vaginal inlay flap.

Tanello et al. described the use of a pedicle flap from the labia minora as treatment for distal stricture in two patients with excellent success.

In one of the largest series, Montorsi et al.used a dorsal approach using vestibular flap with very good result ${ }^{17}$.

So the dorsal approach may preserve the normal anatomic positioning of the urethra upward, whereas ventral approaches tend to redirect the urethral meatus posteriorly; the significance of this in voiding is unclear. Some surgeons have been reluctant to perform dorsal repairs because of concerns for bleeding, damage to clitoral structures, stress incontinence, and neurosensory complications. Therefore, ventral onlay approaches are generally preferred, although the literature is insufficient to support one approach over another.

Considering the outcome and complications of different approaches of urethral reconstructions we prefer to use dorsal approach and dorsal inlay free BMG (buccal mucosal graft) substitution. The intermediate and long term outcome of in our series is excellent as showen in the tables.

So far my knowledge, no publication was yet to be found in our country, Bangladesh to compare my result.

\section{Conclusion:}

The stricture of urethra is not uncommon in our clinical practice. Conventional modalities of urethral stricture management using urethral dilatation, urethrotomy with or without CISC may not be effective in long run and recurrence is common in many of the cases. CISC 
in our socio-cultural context is not feasible in our country, Bangladesh. It needs assistance and frustrating for our patients. However this can be overcome by dorsal substitution urethroplasty using free BMG, in managing female urethral stricture, for long-term recurrence free improved quality of life.It appears that Urethroplasty eliminates or dramatically decreases the number of costly and painful repeat office visits for dilation and urethrotomy.

\section{References}

1. Ackerman AL, Blaivas J, and T. Ange JTFemale Urethral Reconstruction. Curr Bladder Dysfunct Rep. Dec 2010; 5(4): 225-232. Published online Oct 19, 2010. doi: 10.1007/s11884-010-0071-6.

2. Schwender $\mathrm{CE}^{1}, \mathrm{Ng}$ L, McGuire E, Gormley EA. Technique and results of urethroplasty for female stricture disease. J Urol. 2006 Mar;175(3 Pt 1):97680; discussion 980.

3. Chen M. Urethral Strictures in Women. December 18, 2011 Print ArticleCitation ,XMLEmail

4. Farrar DJ, Osborne JL, Stephenson TP, et al. A urodynamic view of bladder outflow obstruction in the female: factors influencing the results of treatment. Br J Urol.1975;47:815-822.

5. Merimsky E. Retention secondary to urethral stricture in the female. Urology.1985;26:598. doi: 10.1016/0090-4295(85)90374-7.

6. Romero Perez P, Mira Llinares A. Renal and ureteral complications of urethral stenosis.Actas Urol Esp. 1995;19:432-440.

7. Santucci RA, Payne CK, Anger JT, Saigal CS. Office dilation of the female urethra: a quality of care problem in the field of urology. J Urol. 2008;180:2068-2075.
8. Indudhara R, Vaidyanathan S, Radotra BD. Urethral tuberculosis. Urol Int.1992;48:436-438.

9. Pugliese JM, Morey AF, Peterson AC. Lichen sclerosus: review of the literature and current recommendations for management. J Urol. 2007;178:2268-2276.

10. Ozel B, Ballard C. Urethral and paraurethral leiomyomas in the female patient. Int Urogynecol J Pelvic Floor Dysfunct. 2006;17:93-95.

11. Cavadas PC, Landin L. Treatment of urethral stricture in a female-to-male transsexual with a tubulized flap from the labia minora. J Reconstr Microsurg. 2005;21:153-156.

12. Santucci RA, Payne CK, Anger JT, Saigal CS. Office dilation of the female urethra: a quality of care problem in the field of urology. J Urol. 2008;180:2068-2075.

13. Schwender CE, Ng L, McGuire E, Gormley EA. Technique and results of urethroplasty for female stricture disease. J Urol. 2006;175:976-980.

14. Female urethral strictures: successful management with long-term clean intermittent catheterization after urethral dilatation. SMITH AL, FERLISE VJ and ROVNER ES, BJU International Volume 98, Issue 1, pages 9699, July 2006.

15. Ngugi PM, Kassim A. Clean intermitent catheterisation in the management of urethral strictures. East Afr Med J. 2007;84:522-524.

16. Stein TA, DeLancey JO. Structure of the perineal membrane in females: gross and microscopic anatomy. Obstet Gynecol. 2008;111:686-693.

17. Montorsi F, Salonia A, Centemero A, et al. Vestibular flap urethroplasty for strictures of the female urethra. Impact on symptoms and flow patterns. Urol Int. 2002;69:12-16. 